\title{
IL DELITTO DELL'ABORTO PROCURATO NELLA CHIESA DELLE ORIGINI
}

\section{INTRODUZIONE}

Ogni uomo, creato ad immagine e somiglianza di Dio, è chiamato a partecipare alla vita dello stesso Dio. La vita umana, essendo un grande dono affidato da Dio all'uomo, ,impone a questi di prendere coscienza del suo inestimabile

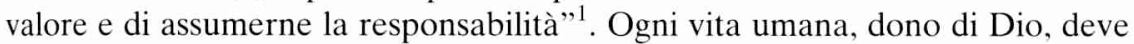
essere, perciò, accolta dall'uomo con riverenza. Infatti, non spetta all'uomo il giudizio sul valore della vita. Esso è stato pronunciato dallo stesso Dio per il fatto di averla chiamata all'esistenza. L'uomo chiamato da Dio alla vita, dal momento del concepimento, rimane unito al Creatore attraverso una peculiare relazione: non porta soltanto in sé la Sua immagine, ma anche, essendo l'unica creatura sulla terra che Dio ha voluto per se stesso, rimane con Lui in uno speciale collegamento ${ }^{2}$. Questa eccezionale relazione tra il Creatore e la creatura, che esige anche necessariamente una santità di vita, chiama l'uomo ad una grande responsabilità verso la vita umana. In questa visione teologica di vita umana l'aborto si presenta non soltanto come una semplice interruzione della vita umana, ma anche come sospensione dell'azione creativa di Dio.

Ciò nonostante, l'uomo molte volte ha dimenticato questa particolare verità $e$, invece di proteggere la vita indifesa dei nascituri, con le sue azioni e con le sue leggi permetteva che questa vita fosse distrutta. La Chiesa, che deve vigilare sulla santità e sull'integrità della vita umana, fin dai primi secoli, con la sua disciplina canonica introduce delle sanzioni penali contro tutti coloro che commettono il delitto di aborto procurato.

L'aborto procurato non è un fenomeno esclusivo dei nostri tempi. Fin dall'inizio del pellegrinaggio umano in questa terra, l'aborto si presenta come uno dei principali delitti contro la vita umana. Questo delitto contro gli umani

${ }^{1}$ Instructio „Donum vitae” de observantia erga vitam nascentem deque procreationis dignitate tuenda. Introductio 1, AAS 80(1988) 71.

${ }^{2}$ Cf. Constitutio „Gaudium et spes” 24, AAS 58(1966) 1045. 
ai quali non è stato permesso di nascere, è stato presente in ogni tempo e in ogni società. Anche l'uomo moderno, che si vanta di tante scoperte a favore della difesa della vita umana, che dice di „sì" alla clonazione delle cellule umane e ai metodi artificiali della concezione, non vuole accettare spesso la vita come tale. Il valore della vita umana nel mondo odierno è compreso molto poco. Seguendo con attenzione le più recenti scoperte scientifiche, si può constatare che l'uomo moderno è giunto ad una situazione assurda. Infatti, volendo ad ogni costo dominare il mistero della vita, dal momento del concepimento fino alla morte, sempre più efficacemente distrugge questa vita, propagando ad ogni costo la cosiddetta ,cultura della morte” che, oltre l'aborto ${ }^{3}$, abbraccia anche l'eutanasia, l'uso delle droghe, l'alcool, il fumo e altre forme di distruzione della vita. La vita umana è stata ridotta a semplice materiale del lavoro umano, e l'homo faber ha occupato il posto del Creatore ${ }^{4}$.

L'aborto presenta oggi caratteri nuovi rispetto al passato. La prima novità è di ordine quantitativo: il numero annuo di aborti a livello mondiale è molto elevato. Ogni anno si praticano oltre cinquanta milioni di aborti. Almeno trenta milioni di essi hanno luogo con il consenso delle leggi di vari Stati. Sono nuove anche alcune circostanze, che caratterizzano l'aborto dal punto di vista qualitativo: la tendenza a presentarlo come se fosse un diritto che lo Stato deve riconoscere e garantire; la sua realizzazione da parte di operatori sanitari, che dovrebbero essere i professionisti della custodia della salute e della promozione della vita; la grave circostanza che tali attentati avvengano spesso all'interno della famiglia ${ }^{5}$. Sfruttando ogni possibile argomento economico, giuridico, politico e sociale, si cerca di cancellare la verità della ,strage contro gli innocenti" che si compie sotto i nostri occhi. Anche parecchi credenti, sotto l'influsso di questa mentalità abortiva, vogliono cambiare la posizione della Chiesa riguardo all'aborto.

Di fronte a questi problemi, ci sembra utile esporre in modo sintetico il pensiero dei Padri della Chiesa e la primissima legislazione della Chiesa riguardo al delitto dell'aborto procurato, contenuta sia nei canoni conciliari, sia nei Libri Penitenziali.

\section{L'ABORTO NEI PADRI DELLA CHIESA}

La problematica riguardante l'aborto nel pensiero dei Padri della Chiesa è inserita nel contesto della proposta di scelta fondamentale di civiltà fatta da Dio

${ }^{3}$ Cf. M. Ryan, Aspetti socio-politici dell'aborto, in: Commento interdisciplinare alla „Evangelium vitae”, ed. Pontificia Accademia per la Vita, Città del Vaticano 1997, 535-548.

${ }^{4}$ Cf. J. Ratzinger, Il fondamento sacramentale dell'esistenza umana, Brescia 1971, 25-28.

5 Cf. A. Rodríguez Luño, L'enciclica „Evangelium vitae” e la tutela giuridica della vita nascente „Identità Cattolica. La vita” 1 (1996) 14. 
al Popolo Eletto: „Scegli la vita, così vivrai tu e la tua discendenza” (Dt 30, 19). Nel periodo apostolico, la prima importante testimonianza è la Didaché. Quest'opera, presentando la „via della vita”, dichiara: ,[...] non ucciderai la prole con aborto né farai perire il nato [...]"6. Chi, dunque, uccide i figli o contamina la creatura di Dio con un aborto, si trova nella „via della morte”. La stessa dichiarazione troviamo nella Lettera dello pseudo Barnaba, dove leggiamo:

„,non ucciderai il concepito con l'aborto" ,,appartiene alla vita della morte [...] chi manda in rovina la creatura di Dio nell'aborto"

I testi sopra indicati, seguendo lo schema della duplice via, insegnano che chi cammina sulla via della vita non uccide e, quindi, non toglie la vita al feto con l'aborto. L'azione abortiva, oltre ad essere una grave mancanza di amore verso il prossimo, è soprattutto un'offesa al diritto di Dio, appunto perché uccide una sua creatura. Il delitto di aborto procurato, secondo un altro passo della Lette$r a$, mette sulla via della morte non soltanto la donna abortista, ma anche chi la fa abortire e conduce, così, tutti e due sulla strada dell'eterno supplizio ${ }^{8}$.

Nell'epoca seguente, gli Apologisti, difendendo la fede e i costumi cristiani, ribadirono che in ogni caso l'aborto è un crimine contro la vita e la santità e come tale viola la dipendenza della vita umana da Dio. Infatti, Atenagora nella Supplica per i cristiani rileva che i cristiani valutano come omicide le donne che usano medicine per abortire; egli condanna chi assassina i bimbi, anche quelli che vivono nel grembo della loro madre, e si ritiene che essi sono già l'oggetto della Provvidenza divina ${ }^{9}$. Atenagora, nella sua opera, indica nello stesso tempo l'importanza del figlio nel matrimonio cristiano e anche la lotta per difendere il diritto alla vita dei figli prima della loro nascita. Parla del feto come di un essere creato, che non può essere ucciso ${ }^{10}$.

L'altro l'apologista, Minucio Felice, nell'Octavius condanna l'aborto come un delitto di omicidio, perché distrugge l'origine di un uomo futuro:

„vi sono anche le donne le quali, bevendo dei medicamenti, distruggono nelle loro viscere l'inizio del futuro uomo e commettono parricidio prima di partorire"11.

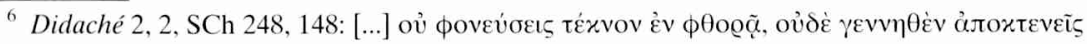
$[\ldots]$.

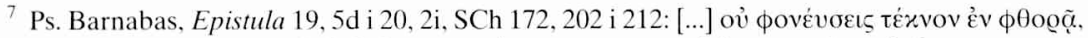

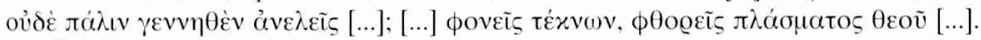

${ }^{8}$ Cf. B. Honings, Aborto, DPAC I 10-11; W. Turek, Gloria Dei vivens homo. I riferimenti patristici dell'Enciclica Evangelium Vitae di Giovanni Paolo II, in: M. Toso (ed.), Educare alla vita, Roma 1996, 78-79. Nell'enciclica il Papa fa riferimento all'insegnamento patristico della Didaché, dell'Apologia per i cristiani di Atenagora e all'A pologetico di Tertulliano.

${ }^{9}$ Cf. Athenagoras, Legatio pro Christianis 35, 5, SCh 379, 204.

${ }^{10}$ Cf. ibidem.

${ }^{11}$ Minucius Felix, Octavius 30, CSEL 2, 43:, ,...] sunt quae in ipsis visceribus, medicaminibus epotis, originem futuri hominis extinguant, et parridicium faciant antequam pariant". 
La parola utilizzata da Minucio parricidium era applicata in questo tempo all'uccisione di un parente, non però del figlio. Utilizzandola, l'autore aveva intenzione di esprimere la gravità ed odiosità che l'aborto procurato aveva per i cristiani, ma purtroppo soltanto per loro, perché di pene comminate dal diritto civile, come al solito, non si fa cenno ${ }^{12}$.

Nella stessa linea pone l'insegnamento nei suoi scritti apologetici Tertulliano. Difendendo nell'Ad nationes i cristiani dall'accusa di infanticidi rituali con antropofagia, dichiara che ai credenti non è lecito praticare l'aborto, perché è un omicidio, e nello stesso tempo accusa i gentili di praticare l'aborto ${ }^{13}$. Il divieto di praticare l'aborto si estende anche all'embrione nell'utero materno. Come afferma Tertulliano nell'Apologeticus ,non fa differenza se uno tolga la vita già nata o la distrugga nascente. Uomo è pure chi lo sarà: «Homo est et qui est futurus»"14.

L'intervento di Tertulliano, riguardo al nostro argomento, non si limita soltanto a queste due opere. Infatti, egli difendeva la vita concepita come dono di Dio, e da Dio sempre protetto, anche nelle altre opere: nel De exhortatione castitatis ${ }^{15}$ e nel De virginibus velandis ${ }^{16}$. La condanna dell'aborto come omicidio in queste opere è molto chiara: non si può mai uccidere la vita, sia già nata, sia non ancora nata, ma già concepita. Negli anni successivi, la posizione di Tertulliano è un po' cambiata. Nella sua opera De anima, affermando l'animazione immediata dell'embrione ${ }^{17}$, parla di una maturazione progressiva dell'anima ${ }^{18}$, insieme con il corpo: solo a maturazione avvenuta si può parlare di homo ${ }^{19}$. Secondo lui, animal il prodotto di concepimento è subito, invece homo diventa dopo un periodo di tempo, cioè ,dopo quel determinato numero di giorni, che la tradizione medico-filosofica del tempo riteneva necessario perché il feto potesse dirsi «formato»: significativamente Tertulliano si appella qui all'Es 21, 22-25 nella versione dei Settanta, sottolineando che la pena di morte è ivi stabilita solo per l'aborto di un feto "formato" " 20 .

${ }^{12}$ Cf. E. Nardi, Procurato aborto nel mondo greco-romano, Milano 1971, 393; P. Sardi, L'aborto ieri e oggi, Brescia 1975, 69-70. Così anche J. Palazzini: „Nec minus aperte hoc crimen damnat Minucius Felix, qui imperante Caracalla vixit, iuris romanis scientiae haud ignarus". (Ius fetus ad vitam eiusque tutela in fontibus ac doctrina canonica usque ad saeculum XVI, Romae 1943, 44).

${ }^{13}$ Cf. Tertullianus, Ad nationes I 15, 8, CCL 1, 34.

${ }^{14}$ Idem, Apologeticus 9, 8, CCL 1, 103.

15 Cf. Idem, De exhortatione castitatis 12, CCL 2, 1032-1033.

16 Cf. Idem, De virginibus velandis 14, CCL 2, 1223-1224.

17 Cf. Idem, De anima 25; 27; 36, CCL 2, 819-821, 822-824, 838-839.

18 Cf. ibidem, 37, CCL 2, 839-840.

19 Cf. ibidem.

20 P. Sardi, L'aborto ieri e oggi, art. cit., 71. Cf. E. Sgreccia, L'insegnamento dei Padri della Chiesa, in: A. Fiori - E. Sgreccia (ed.), L'aborto. Riflessioni di studiosi cattolici, Milano 1975, 56: J. Palazzini, Ius fetus ad vitam eiusque tutela in fontibus ac doctrina canonica, op. cit., 43. Come 
L'altro problema che viene affrontato da Tertulliano nel De anima è la così detta l'embriotomia terapeutica. L'autore prende in considerazione il caso di una gravidanza, che risulta pericolosa per la vita della madre e che non può essere portata a termine senza che la madre muoia. In questa situazione l'autore segue l'opinione dei medici che in questo caso fanno l'embriotomia. La situazione è caratterizzata dalla crudeltà, ma come afferma di una necessaria crudelitas, perché se il feto non muore diventa marticida. Tertulliano in questo caso non pronuncia alcuna condanna, ma si dimostra permissivo ${ }^{21}$. Come abbiamo visto, il pensiero di Tertulliano è un po' sfumato. Infatti, egli afferma con chiarezza che l'aborto è un omicidio ed è vietato, ma - come menziona $\mathrm{P}$. Sardi - ,il «no» all'aborto non è così netto come negli autori precedenti, e cominciano a delinearsi delle eccezioni" 22 .

Molto chiaro è invece, nel suo insegnamento, Clemente di Alessandria. L'aborto procurato è contrario alla natura e all'umanità, perché il feto è un essere vivente. La vita umana, sin dall'inizio, si trova sotto la protezione della divina provvidenza, perciò non può essere uccisa ${ }^{23}$.

L'altro testo, più polemico che apologetico, che difendeva la posizione della Chiesa riguardo all'aborto, è la Confutazione di tutte le eresie di Ippolito Romano. Prendendo in considerazione vari problemi morali e dottrinali, egli polemizza con papa Callisto e lo accusa di lassismo non soltanto verso i lapsi ma anche verso i cristiani, che erano colpevoli di vari peccati, anche di aborto. Responsabile di questa situazione era papa Callisto, perché egli aveva incoraggiato le donne, mentre le avrebbe dovuto colpire con la scomunica, come si usava fare con le persone ree di omicidio ${ }^{24}$.

La posizione di Cipriano, vescovo di Cartagine, riguardo al nostro argomento, ci offre varie testimonianze, non solo dei problemi morali connessi con l'aborto, ma anche le testimonianze in materia giuridica. Egli, infatti, informando in una lettera papa Cornelio circa il caso di un suo prete, Novato, che durante il calcio aveva provocato l'aborto, considera quel fatto omicidio. Il prete colpevole dovrebbe essere rimosso dal sacerdozio e scomunicato dopo un processo; ma, a causa della persecuzione di Decio, il processo era stato bloccato: „Onde riteneva per certo che non solo sarebbe stato rimosso dal sacerdozio, ma anche scomunicato $[\ldots]^{\prime 25}$. Come afferma E. Nardi, questo

afferma P. Sardi, il cambiamento di pensiero di Tertulliano non è per tutti gli autori interpretato in modo identico.

${ }^{21}$ Cf. Tertullianus, De anima 25, CCL 2, 836-838. Cf. B. Honings, Aborto e animazione umana, Roma 1973, 71; P. Sardi, L'aborto ieri e oggi, op. cit., 71.

22 P. Sardi, L'aborto ieri e oggi, op. cit., 71.

23 Cf. Clemens Alexandrinus, Paedagogus I 35, SCh 70, 174.

${ }^{24}$ Cf. Hippolytus Romanus, Refutatio omnium haeresium IX 12, 25, GCS 26, 250-251.

25 Cyprianus, Epistula 52,3, CCL 3B, 248: „Propter hoc se non de presbyterio excitari tantum, sed et communicatione prohiberi pro certo tenebat $[\ldots]$. 
documento ha tanta importanza. „É un documento da cui risulta che, anche per quel fatto, il prete colpevole doveva subire un processo davanti al vescovo, e che, anche per quello, e forse per quello prima di tutto, sapeva di doversi aspettare la privazione del sacerdozio e la scomunica" $" 26$.

Grande importanza, riguardo al nostro argomento, aveva la dottrina di San Basilio. Rispondendo ai dubbi presentati dal vescovo Anfilochio di Iconio, egli mostra che la sua dottrina non è differente dai canoni della Chiesa. Egli rifiuta la distinzione tra il feto formato e non formato, considerando tale distinzione come una sottigliezza dei filosofi e dei dotti pagani, che presso i cristiani non si accet$\mathrm{ta}^{27}$. L'aborto come soppressione violenta di una vita umana è sempre un omicidio e come tale deve essere punito. La penitenza, secondo San Basilio, non deve essere prolungata fino alla fine, ma può durare dieci anni, perché non è importante il tempo ma il modo del pentimento. La qualifica di omicida è riconosciuta anche alla donna che propone e che accetta i veleni che uccidono i feti ${ }^{28}$.

Come abbiamo visto, secondo San Basilio, non esiste differenza tra il feto formato e non formato, l'aborto è sempre un omicidio ${ }^{29}$. Una posiziona un po' diversa presenta Teodoreto di Ciro nelle Questioni sull'Esodo. Infatti, ritiene che, avendo una donna abortito in rissa, se il feto venga formato, il fatto si consideri omicidio e il colpevole subisca la stessa pena; se invece venga fuori non formato, non sia ritenuto omicidio, dato che era stato espulso non ancora animato; ma che il colpevole paghi una penale ${ }^{30}$. Secondo quest'opinione, l'aborto di un feto formato è omicidio e come tale deve essere punito con la morte, invece l'aborto di un feto non formato non si può considerare omicidio e viene punito con una multa. Questo non significa che l'autore tratti come lecito l'espulsione del feto non formato; secondo lui , questo caso non ha ancora la qualifica vera e propria di «omicidio», rimanendo sempre, però, colpa e colpa da punire" 31 .

In modo più ampio presenta la sua posizione Sant'Agostino. Il problema dell'aborto viene da lui trattato quando prende in considerazione l'animazione del feto, i diritti del feto e la sorte eterna di esso. L'illiceità dell'atto abortivo in qualsiasi momento della vita del feto umano viene sottolineata, tra l'altro, nel

26 E. Nardi, Procurato aborto nel mondo greco-romano, op. cit., 466-467. Così anche J. Palazzini: ,Cum S. Cypriano parum in legislatione Ecclesiae tertii saeculi penetramus, in eius systema poenale et in actionem prosequendam. [...] Ex his deducimus: 1) Ecclesiam solere ad suum tribunal revocare inter alia etiam abortus crimen. 2) Pro hoc crimine speciales poenas applicari. Duplex hic poena memoratur: degradatio et excommunicatio". (Ius fetus ad vitam eiusque tutela in fontibus ac doctrina canonica, op. cit., 45-46).

27 Cf. Basilius, Epistula 188, 2, PG 32, 671.

${ }^{28}$ Cf. ibidem.

${ }^{29}$ Questa dottrina viene anche confermata da Gregorio di Nissa. Cf. Gregorius Nyssenus, De hominis opificio 29, PG 44, 234-235; SCh 6.

${ }^{30}$ Cf. Theodoretus Cyrensis, Quaestiones in Exodum 21, 48, PG 80, 271-274.

31 E. Sgreccia, L'insegnamento dei Padri della Chiesa, op. cit., p. 60. 
De nuptiis et concupiscentia ${ }^{32}$ e nell'Enarrationes in Psalmos ${ }^{33}$; l'aborto è la distruzione dell'opera di Dio nel grembo materno.

Il passo di Sant'Agostino più commentato è quello che si riferisce all'opera Quaestiones in Heptateuchum, II: quaestiones Exodi LXXX. Come è stato già accennato, il testo dei Settanta fa distinzione tra il feto formato e l'informe. Basandosi su di essa, egli afferma:

„quel che non è formato, non possa neanche intendersi animato, e quindi non sia omicidio, [...] per ciò la legge non volle che il caso attenesse all'omicidio, in quanto non ancora può dirsi viva l'anima in quel corpo che privo di senso, seppure un'anima ci sia in carne ancora informe, e pertanto non ancor dotata di sensi ${ }^{34}$.

Come nota E. Sgreccia, in questo brano l'autore ,non intende pronunciarsi alla leggera sulle questioni dell'animazione, ma questa affermazione non va disgiunta dai brani dello stesso Sant'Agostino"35. Egli non dubita sulla illeceità della espulsione del feto, né rimane incerto sulla qualifica di tale peccato. L'incertezza circa la vera e propria qualifica di omicidio, rimane nella questione relativa all'animazione e nel testo dell'Esodo dei Settanta. Rimane, comunque, fuori discussione, in Sant'Agostino, la illeceità dell'aborto in qualsiasi momento della vita del feto.

Il problema dell'aborto viene affrontato anche nell'Enchiridion. Analizzando la questione della risurrezione dei feti abortivi anche non formati, Agostino ipotizza la possibilità che per essi si verifichi un completamento ad opera della risurrezione:

„Ma chi oserebbe negare, anche se affermare non oserebbe, che la risurrezione operi nel senso di completare ciò che nella forma mancò?"36.

Come si vede, l'autore non esclude la possibilità della risurrezione. L'unico motivo, che giustificherebbe la risurrezione, sarebbe fatto che gli esseri non abbiano avuto, al momento dell'aborto, una loro vita propria. Ma qui, come

32 Cf. Augustinus, De nuptiis et concupiscentia I 15, 17, PL 44, 423-424.

33 Cf. Idem, Enarrationes in Psalmos 57, 5, CCL 39, 712-713.

34 Cf. Idem, Quaestiones in Heptateuchum II 80 (In Exodum 21, 22-25), PL 34, 626: „,...] utrum quod formatum non est, ne animatum quidem possit intellegi, et ideo non sit homicidium [...]. [...] ideo lex noluit ad homicidium pertinere, quia nondum dici potest anima viva in eo corpore quod sensu caret, si talis est in carne nondum formata, et ideo nondum sensibus praedita".

35 E. Sgreccia, L'insegnamento dei Padri della Chiesa, op. cit., 63. L'autore fa anche riferimento al testo di J. Palazzini, Ius fetus ad vitam eiusque tutela in fontibus ac doctrina canonica, op. cit., 75.

36 Augustinus, Enchiridion 85, PL 40, 272: ,,Sed quis negare audeat, etsi affirmare non audeat, id acturam resurrectionem, ut quidquid formae defuit impleatur?". Il problema della risurrezione va accennato anche nel De civitate Dei, dove Agostino non fa distinzione tra i feti formati e gli informi, e dirà di non vedere per qual motivo non dovrebbero risorgere i feti morti nell'utero prima di nascere. Cf. Augustinus, De civitate Dei XXII 13, CCL 48, 833. 
osserva P. Sardi, si trova il punto oscuro: quando il feto comincia a vivere? La risposta a questa domanda, secondo l'autore, è stata data anche da Agostino: „potrebbe infatti esservi nel feto una vita segreta che non si rivela con alcun movimento" 37 .

\section{LE PRIME LEGISLAZIONI CONCILIARI}

Dopo tanti anni d'insegnamento cristiano sul valore della vita umana, l'argomento dell'aborto procurato comincia ad essere oggetto del magistero ufficiale della Chiesa, cioè dei canoni dei concili. Anche se non esiste unanimità tra gli autori a proposito del perché solamente nel IV secolo i concili siano occupati dell'aborto $^{38}$, bisogna dire che i canoni dei concili sia provinciali che universali, iniziarono a regolare validamente non soltanto le questioni disciplinari o penitenziali, ma anche quelle morali. Così in proposito afferma B. Honings: „il giudizio morale sull'aborto non presenta un colore locale, ma è veramente universale. Poiché, anche se la penitenza si sposta da un estremo rigorismo ad una mitigazione, essa è sempre tale, da far capire la gravità del delitto" 39 .

La posizione della Chiesa riguardo all'aborto è stata sempre invariata; esso è stato sempre condannato con gravi pene stabilite dai primissimi concili. Il primo concilio cristiano, che affronta il problema dell'aborto, è stato quello di Elvira in Spagna, celebrato intorno al 305 d. C. Infatti, i suoi due canoni 63 e 68 toccano direttamente il caso di aborto compiuto da una donna, sia cristiana che catecumena:

„Si qua mulier per adulterium, absente marito, conceperit, idque post facinus occiderit, placuit ei neque in fine dandam esse communionem, eo quod geminaverit scelus" ${ }^{40}$.

Invece, riguardo ad una catecumena, il canone stabilisce: „Catechumena si per adulterium conceperit et praefocaverit, placuit eam in fine baptizare" 41 .

I canoni sopra nominati vengono per vari autori ${ }^{42}$ indicati come precedenti ai canoni del sinodo di Ancira, ma secondo P. Sardi questa tesi è contestabile

37 P. Sardi, L'aborto ieri e oggi, op. cit., 91.

38 Cf. E. Nardi, Procurato aborto nel mondo greco-romano, op. cit., 484-486. Secondo l'autore i canoni erano necessari in questo tempo perché con la conversione dei pagani al cristianesimo, loro spesso portarono nella Chiesa i costumi della società pagana che devono essere eliminati.

39 B. Honings, Aborto e animazione umana, op. cit., 103.

${ }^{40}$ Concilium Eliberitanum, can. 63, Mansi II 16.

41 Ibidem, can. 68, Mansi II 17.

42 Cf. F. X. Wernz - P. Vidal, Ius canonicum. Ius poenale ecclesiasticum, VII, Roma 1937, 516, nota 36; J. Palazzini, Ius fetus ad vitam eiusque tutela in fontibus ac doctrina canonica usque ad saeculum XVI, op. cit., 42 e 48; P. Ciprotti, Aborto-Diritto canonico, in: Enciclopedia del Diritto, I, Milano 1958, 140. 
per due ragioni. La remissione della scomunica, secondo i canoni di Elvira, non è contemplata neppur per il termine della vita, invece il Concilio di Ancira, come vedremo, afferma che la norma precedente allontanava le colpevoli dalla comunione soltanto fino al termine della vita; tutto lascia pensare che i canoni non parlano di aborto, ma di infanticidio, ed i termini richiamano espressamente l'uccisione del bambino già nato ${ }^{43}$.

Il Concilio orientale di Ancira, del 314, presenta una disciplina molto peculiare: stabilisce una penitenza temporale per il crimine di aborto. Infatti, il can. 21 afferma:

„De mulieribus quae fornicantur et partus suos necant, sed et de his qui agunt ut utero conceptum excutiant, antiqua quidem definitio usque ad exitum vitae eas ab Ecclesia removet; humanius autem nunc definimus, ut eis decem annorum tempus poenitentiae tribuatur secundum praefixos gradus" ${ }^{24}$.

Secondo questo canone, l'aborto è stato assimilato all'infanticidio, la pena prevista per questi delitti è la scomunica di dieci anni e divisa in vari gradi di penitenza pubblica. Ma, com'è stato già indicato tante volte, la dottrina del tempo ha sempre affermato che l'aborto è un omicidio: i canoni Ancirani, can. 22 che parla di omicidio volontario e il can. 23 che parla di omicidio involontario $^{45}$, non fanno alcun riferimento a questa dottrina. Secondo P. Sardi „tale diversità pare doversi attribuire alla diversa esteriore apparenza del delitto, alle attenuanti che spesso lo accompagnano e, infine, al minor danno sociale che in genere deriva dall'aborto-infanticidio rispetto all'omicidio di persona adulta" 46 .

Troviamo il divieto di aborto anche nei Canones Ecclesiastici Sanctorum Apostolorum. Il sesto canone di quest'opera in modo ben determinato vieta di uccidere la prole con l'aborto ${ }^{47}$. Negli anni successivi la penitenza prevista per il delitto di aborto procurato è stata mutata. Infatti, nel 524, il Concilio pro-

${ }^{43}$ Cf. P. Sardi, L'aborto ieri e oggi, op. cit., 76. Così anche J. Palazzini: „Tamen non omnino elucet utrum de abortu an de infanticidio Concilium loquatur in canonibus 63 et 68 " (Ius fetus ad vitam eiusque tutela in fontibus ac doctrina canonica usque ad saeculum XVI, op. cit., 49). La stessa opinione condivide anche E. Nardi, Procurato aborto nel mondo greco-romano, op. cit., 490-491.

${ }^{44}$ Concilium Ancyranum, can. 21, Mansi II 519. La norma antica alla quale fa riferimento il canone, come è stato già accennato, non è il Concilio di Elvira, ma può corrispondere con la disciplina dell'epoca di papa Callisto e di Cipriano. Cf. E. Nardi, Procurato aborto nel mondo greco-romano, op. cit., 497-498; P. Sardi, L'aborto ieri e oggi, op. cit., 77.

${ }^{45}$ Concilium Ancyranum, can. 22-23, Mansi II 519-522.

46 P. Sardi, L'aborto ieri e oggi, op. cit., 77. Nella stessa linea si trova anche J. Palazzini: „Diferentia si adest, est in gradu, eo quod in abortu saepe minus habetur damnum sociale quam in homicidio. Exinde explicatur quomodo etiam in iure canonico paulatium facta est differentia in applicandis poenis". (Ius fetus ad vitam eiusque tutela in fontibus ac doctrina canonica, op. cit., 52).

${ }^{47}$ Cf. Canones Apostolorum ecclesiastici, can. 6, in: F.X. Funk (ed.), Doctrina duodecim Apostolorum. Canones Apostolorum ecclesiastici, Tubingae 1887, 52. 
vinciale a Lerida ha ridotto la penitenza di dieci anni a sette, con la clausola „rei omni tempore vitae suae fletibus, et humilitati insistant" ${ }^{48}$. Il Concilio ha previsto anche la pena peculiare per i chierici: „Si vero clerici fuerint, officium eis ministrandi recuperare non liceit; attamen in choro psallentium a tempore receptae communionis intersint ${ }^{\text {"49 }}$. La pena più severa, invece, è stata prevista per gli spacciatori di veleni: una scomunica da rimettersi solo in punto di morte $^{50}$.

Un particolare iter presenta il III Concilio di Toledo, celebrato nel 589. Infatti, esso rappresenta la collaborazione tra sacerdoti e giudici laici per combattere il delitto di aborto procurato, che si è stato diffuso in alcune parti della Spagna. Come rimedio il Concilio stabilisce: „capitali vindicta acriori disciplina prohibeant" ${ }^{\prime 51}$.

I vari concili di Gallia celebrati in questo periodo, trattando il problema della scomunica prevista per l'omicidio, non stabiliscono il tempo della penitenza $^{52}$, in alcuni casi il giudizio di stabilire il modo penitenziale è lasciato al sacerdote.

Al tempo di dieci anni di penitenza previsti per l'aborto torna il Concilio di Trullo, celebrato nel 692 a Costantinopoli. Il Concilio afferma che l'aborto è un omicidio e come tale deve essere punito. La pena non è stata stabilita espressamente, ma, avendo fatto riferimento alla dottrina precedente, sia al Concilio di Ancira che al pensiero greco, si intende che la sanzione sia la scomunica ${ }^{53}$. Il canone mette sullo stesso piano le donne che fanno uso dei veleni abortivi e coloro che ,dant abortionem facientia medicamenta" $" 54$.

Anche i concili celebrati in Germania hanno fatto riferimento alla normativa precedente. Infatti, il can. 21 del I Concilio di Magonza del 847 riproduce il can. 21 di Ancira: ,decem annorum tempus secundum praefixos gradus poenitentiae largiatur" ${ }^{55}$. Poi, citando il can. 63 di Elvira riguardante il doppio

48 Concilium Ilerdense, can. 2, Mansi VIII 612.

49 Ibidem.

50 Cf. J. Palazzini, Ius fetus ad vitam eiusque tutela in fontibus ac doctrina canonica, op. cit., 79: „Ut delictum radicitis pro amoveatur maior poena pro veneficis statuitur, videlicet pro iis qui venea, inter quae lex romana etiam venena ad prolem interficiendam tendentia continebat, conficiunt et vendunt".

51 Concilium Toletanum III, can. 17, Mansi IX 997.

52 Così per esempio il Concilio di Tours, di Vanes, di Agde. Cf. B. Honings, Aborto e animazione umana, op. cit., 111.

53 Cf. Concilium Trullanum, can. 91, Mansi XI 982. Cf. anche S. Marcilla Catálan, El delito canónico de aborto: praxis sacramental y abolición de la censura, I, „Mayéutica” 20 (1994) 150. L'autore, presentando la legislazione di questo concilio greco osserva che, mentre il diritto romano si occupava soltanto di aborto fatto dalla donna sposata, il diritto bizantino per proteggere la vita del feto prendeva in considerazione anche le donne non sposate.

${ }^{54}$ Cf. Concilium Trullanum, can. 91, Mansi XI 982.

55 Concilium Moguntinum, can. 21, Mansi XIV 909. 
peccato di adulterio e di aborto, è stata mitigata la penitenza, che ammette la scomunica di sette anni ${ }^{56}$. Di nuovo il problema dell'aborto procurato viene trattato dal Concilio di Worms del 868. Confermando che l'aborto è un omicidio, il can. 35 prende in considerazione il caso di aborto e il caso di infanticidio per soffocamento durante il sonno; ogni caso deve essere giudicato diversamente:

„Mulieres igitur quae ante temporis plenitudinem conceptos utero infantes voluntate excutiant, ut homicidae procul dubbio iudicandae sunt. Illae vero quae dormiendo filios suffocare videntur, leviter de his iudicare oportet $"$ "57.

\section{I LIBRI PENITENZIALI}

Nel corso del VI secolo, sotto l'influenza delle pratiche proveniente dal mondo insulare, si sviluppa nella Chiesa una nuova disciplina penitenziale, penitenza tariffata o tassata che consiste nella precisa determinazione della pena $^{58}$. I Libri Paenitentiales come indici o tavole di vizi con l'indicazione della rispettiva penitenza ecclesiastica da imporre ai sacerdoti per la riconciliazione del penitente, contengono anche la materia che riguarda il nostro argomento. I delitti contro la vita umana, incluso l'aborto, sono collocati nei Libri Penitenziali: ,a) inter crimina homicidi; b) quandoque vero in materia de VI praecepto vel in iure matrimoniali; c) interdum inter malificii delicta"59.

Il primo dei penitenziali, che in modo diretto fa riferimento al nostro argomento, è il Poenitentiale Vinniani che stabilisce la pena per la malefica: digiuno per mezzo anno a pane e acqua, astensione dal vino e dalla carne per due anni, digiuno a pane e acqua per sei quaresime $e^{60}$.

${ }^{56}$ Cf. ibidem, can. 21, Mansi XIV 909. Secondo B. Honings, la ragione per cui il concilio tedesco non ha deciso tassativamente d'imporre la penitenza agli abortisti, può essere che „il concilio, ispirato ormai al principio modale e non cronologico della sanzione, ha stigmatizzato la gravità del delitto, ma, nello stesso tempo, ha lasciato al giudizio del sacerdote confessore di determinare la corrispondente penitenza". Secondo l'autore, questa tesi con limiti della sua probabilità è stata confermata con la legislazione del Concilio di Worms (Cf. Aborto e animazione umana, op. cit., 115).

57 Concilium Wormatiense, can. 35, Mansi XV 876. In modo più ampio con riferimento ai altri concili tratta B. Honings, Aborto e animazione umana, op. cit., 115-116.

58 Sulla pena e la penitenza proposta dai Libri Penitenziali, cf. B. Honings, Aborto e animazione umana, op. cit., 116-122; Idem, L'aborto nei Libri Penitenziali irlandesi, „Apollinaris” 48 (1975) 501-507; A. Di Berardino, Letteratura canonica, penitenziale e liturgica, in: Patrologia, IV, Genova 1996, 514-522; P. Sardi, L'aborto ieri e oggi, op. cit., 108-110; B. E. Ferme, Introduzione alla storia delle fonti del diritto canonico, I, Roma 1998, 105-112.

59 J. Palazzini, Ius fetus ad vitam eiusque tutela in fontibus ac doctrina canonica, op. cit., 90-91; M. G. Muzzarelli, Il valore della vita nell'alto medioevo: la testimonianza dei Libri Penitenziali, „Aevum” 62 (1988) 171-185.

${ }^{60}$ Cf. Poenitentiale Vinniani 20, in: F.W.H. Wasserchleben, Die Bussordungen der abendländischen Kirche, nebst einer rechtsgeschichtlichen Einleitung, Halle 1851, 112. 
Nella stessa linea colloca l'aborto il De poenitentiarum mensura taxanda, composto da San Colombano. Facendo distinzione tra il melefico mortifero e quello amoroso, San Colombano fa differenza anche nella penitenza. Nel primo caso la penitenza consiste nei tre anni di digiuno a pane e acqua, più tre anni di astinenza dal vino e dalla carne. Nel secondo caso, invece, fa distinzione riguardo dell'autore del delitto: laico, semplice chierico, diacono e sacerdote, e stabilisce la penitenza di un anno di digiuno a pane e acqua per il chierico, la stessa penitenza ma soltanto per un mezzo anno per il laico, e alla fine due o tre anni per un diacono o sacerdote ${ }^{61}$.

Il Poenitentiale Cummeani parla di aborto tante volte: sotto la rubrica de adulterio, raptu et incensu, poi de homicidio et sanguinis effusione e, infine, de maleficis et veneficis. La penitenza prevista per l'aborto è, come nel caso di Ancira, dieci anni:

„Mulieres quae fornicantur et partus suos necat, sed et si quid agant secum ut uteros conceptos discutiant, $\mathrm{X}$ annos poeniteant" ${ }^{\mathrm{62}}$.

Questa posizione poi è stata cambiata: un anno, se si tratta di aborto di feto animato, cioè entro 40 giorni dal concepimento, tre anni invece se l'animazione era già avvenuta, trattandosi in questo caso di omicidio ${ }^{63}$. Il penitenziale fa anche distinzione tra l'omicidio volontario e quello casuale e, in base a ciò, stabilisce la penitenza da imporre al colpevole, prendendo in considerazione - come nel caso di San Colombano - anche lo stesso autore del delitto $^{64}$. La penitenza di tre anni per l'aborto volontario propone il Poenitentiale Merseburgense ${ }^{65}$, il Poenitentiale Parisiense ${ }^{66}$ e il Poenitentiale Vindobonense ${ }^{67}$.

${ }^{61}$ Cf. Columbanus Hibernus, De poenitentiarum mensura taxanda 18, PL 80, 226.

${ }^{62}$ Cummianus Hibernus, De mensura poenitentiarum 3, PL 87, 987.

${ }^{63}$ Cf. ibidem, 6, PL 87, 989-990; P. Sardi, L'aborto ieri e oggi, op. cit., 111. La riduzione della penitenza viene giustificata in base alla maggior durezza delle penitenze stabilite per Cummeano.

${ }^{64}$ Il Penitenziale di Cummeano fa distinzioni più numerevoli rispetto al Penitenziale di Colombano. Si noti il maggior rigore nei confronti del diacono e del sacerdote. La differenza, secondo B. Honings, dipende dal fatto che „Cummeano mette tra il chierico e il diacono, che aveva due anni nel penitenziale di Colombano, il suddiacono e così la penitenza del diacono passò al subdiacono, esigendo l'aumento per il diacono; altretanto si dice per il sacerdote". (Aborto e animazione umana, op. cit., 130). Cf. anche idem, L'aborto nei Libri Penitenziali irlandesi, op. cit., 513-514.

65 Cf. Poenitentiale Merseburgense 33, in: H. J. Schmitz, Die Bussbücher und Bussdisziplin der Kirche, II, Mainz $1883^{2}, 362$.

${ }^{66}$ Cf. Poenitentiale Parisiense 27, in: H. J. Schmitz, Die Bussbücher und Bussdisziplin der Kirche, op. cit., 329.

${ }^{67}$ Cf. Poenitentiale Vindobonense 36, in: H. J. Schmitz, Die Bussbücher und Bussdisziplin der Kirche, op. cit., 353. 
Il nuovo modo di vedere la problematica dell'aborto presenta il Poenitentiale di Pseudo-Teodoro che fa la distinzione tra il feto animato e quello non ancora animato:

„Mulier sponte perdens partum suum, si ante 40 dies conceptionis, annum poeniteat, si vero post, tres annos poeniteat" ${ }^{168}$.

Secondo lui, se la perdita del feto avviene dopo i 40 giorni, l'azione volontaria che ne è la causa è abortiva, e la penitenza prevista sono tre anni di digiuno. Se, invece, la perdita avviene prima dei 40 giorni, la penitenza è limitata ad un anno di digiuno. Questo significa che, anche se il feto non ha ancora raggiunto il tempo richiesto per il primo movimento, si tratta di un'azione moralmente reprensibile ${ }^{69}$.

La stessa penitenza propone anche il Poenitentiale del Venerabile Beda. Egli ha unito i casi dell'aborto e dell'infanticidio e li ha collocati nel capitolo $D e$ occisione:

„Mulier quae occidit filium suum in utero ante XL dies, I annum poeniteat. Si vero post XL dies, III annos. Sed distat multum, utrum paupercula pro difficultate nutriendi an fornicaria causa sui sceleris celandi faciat" ${ }^{270}$.

Come abbiamo visto, i Libri Penitenziali provenienti da varie famiglie sempre giudicano il delitto di aborto procurato omicidio. Anche se le penitenze proposte prima affermavano le norme penitenziali dei concili e poi sono cambiate $^{71}$, la cosa più importante è che la ferma dottrina della Chiesa riguardo all'aborto non è stata mai mutata ${ }^{72}$.

Anche le varie leggi delle popolazioni barbariche hanno considerato l'aborto come un delitto ${ }^{73}$, ma soltanto a motivo del danno provocato al marito

${ }^{68}$ Ps. Theodorus Cantuariensis, Poenitentiale 77, PL 99, 957.

${ }^{69}$ Cf. B. Honings, Aborto e animazione umana, op. cit., 134-135.

70 Ps-Beda Venerabilis, Poenitentiale 12, in F.W.H. Wasserschleben, Die Bussordungen der abendländischen Kirche, op. cit., 225.

71 Secondo P. Sardi, le varie norme proposte dai Penitenziali non davano al confessore un criterio chiaro da applicare: „La conseguenza è che l'arbitrio dei singoli confessori nell'applicare le penitenze può sbizzarrirsi a piacimento". Il lassismo in questa materia conduceva ad applicare le penitenze, che erano impossibili da compiere, ma la situazione poi è stata cambiata. „Questa situazione di lassismo e di confusione era la prova evidente che i penitenziali avevano fallito lo scopo per il quale erano sorti, quello cioè di mettere ordine nell'amministrazione della penitenza". (L'aborto ieri e oggi, op. cit., 115-116).

${ }^{72}$ M. G. Muzzarelli così conclude sul valore della vita nei Libri Penitenziali: „Sembra invece possibile asserire che nel nome della assoluta autorità di Dio sugli uomini la Chiesa altomediovale ha mortificato la libertà del singolo ma ha certamente difeso il diritto alla vita anche dei più deboli. Una pagina importante su una fase che definirei decisiva della storia evolutiva della nostra civiltà occidentale sta scritta proprio nei libri penitenziali". (Il valore della vita nell'alto medioevo: la testimonianza dei Libri Penitenziali, op. cit., 185).

73 In modo dettagliato presenta la normativa delle leggi barbariche J. Palazzini, Ius fetus ad vitam eiusque tutela in fontibus ac doctrina canonica, op. cit., 81-89. 
o al padrone o alla donna stessa ${ }^{74}$. Le sanzioni previste consistevano nel pagamento di una somma come ricompensa per quel danno. Il primo documento, che fa riferimento diretto al feto e alla sua vitalità è il Pactus Legis Almannorum:

„,Si quis mulier gravata fuerit et per facto alterius infans mortuus natus fuerit et aut si vivus natus fuerit et IX noctis non vivit, cui reputatum fuerit, XL solidos componat" ${ }^{\mathrm{75}}$.

Invece, una prima forma di pena vendicativa, che persegue il crimine dell'aborto per se stesso, si trova nella Lex Visigothorum, e punisce con la pena di morte chi ha fornito la pozione velenosa; la donna schiava deve essere sottoposta a duecento colpi di flagello, la libera viene ridotta in stato di schiavitù $^{76}$.

Grazie all'influsso dei principi cristiani, la legislazione dei popoli barbari ha subito un cambiamento. Le leggi proteggono in modo più significativo il feto e la sua integrità fisica, ma non, come era prima, il diritto del padre o del padrone. La pena prevista per la madre o per il padre colpevoli di questo crimine, è la morte o l'accecamento ${ }^{77}$. L'influsso del pensiero cristiano si nota anche nella normativa proposta nel Liber Papiensi. Commentando l'Edicto Rothari, che ha considerato soltanto i casi di aborto causato involontariamen$\mathrm{te}^{78}$, stabilisce che „quoniam hec lex facit puerum componi, quando in utero matris nolendo occisus est, multo melius debemus intelligere, quod debet componi, quando in utero matris occisus est volendo",79.

La legislazione dei primi concili, che abbiamo già esaminato, è stata di nuovo confermata nel VIII sec., nelle collezioni canoniche. Infatti, la Collectio Dacheriana, affrontando nel primo libro il tema della penitenza, menziona esplicitamente l'aborto, per il quale viene citato il can. 21 del Concilio di Ancira ${ }^{80}$. Anche la Collectio Pseudo-Isidoriana, benché si riferisca più all'in-

${ }^{74}$ Così Pactus Legis Salicae, Lex Salica, Lex Ribuaria, dove l'aborto viene punito per danno arrecato alla donna. Cf. J. Palazzini, Ius fetus ad vitam eiusque tutela in fontibus ac doctrina canonica, op. cit., 81-82; P. Sardi, L'aborto ieri e oggi, op. cit., 117.

${ }^{75}$ Pactus Legis Almannorum, ex fragmentis Codicis $A$, fragmentum secundum, 31, ed. J. Merkle, MGH III 35.

76 Cf. Lex Visigothorum 6, 3, 1, ed. K. Zeumer, MGH Leges I 1, 260-262.

77 Cf. ibidem.

78 Cf. Edictum Rothari 75 e 334, ed. F. Bluhme, MGH Leges IV 24 e 76.

79 Liber Papiensis, riportato nell'edizione citata dell'Edicto Rothari al n. 75, 5, MGH Leges IV 308.

${ }^{80}$ Cf. Collectio Dacheriana 1, 56, 100, in: L. D'Achery, Veterum aliquot scriptorum qui in Galliae Bibliothecis, maxime Benedictorum latuerant spicilegium, I, Parisiis $1672^{2}, 525-530$. Dopo la riforma carolingia sono apparsi di nuovo vari penitenziali che ripetono la normativa dei concili, soprattutto di Ancira, o stabiliscono un altro tempo di penitenza, p.e. il Burcardo nel suo Decretum cita i primi concili sottolineando la penitenza che dura dieci anni quale indice della mitigazione per 
fanticidio che all'aborto, riporta i canoni dei tre concili antichi, cui aggiunge anche il can. 17 del III Concilio di Toledo ${ }^{81}$.

Il problema dell'aborto procurato e le conseguenze giuridiche di questo crimine si sono sviluppate nell'ambiente cristiano sia latino sia greco. La Chiesa in questo periodo unanimemente afferma che l'aborto, in ogni caso, è un omicidio e come tale deve essere punito.

Al tempo dei Padri della Chiesa, il giudizio morale sull'aborto è fortemente negativo, perché viene trattato come omicidio e come tale punito severamente. Anche se i primi scrittori non fanno distinzione tra feto formato o non formato, con Tertulliano comincia ad esistere un'interrogativo sul momento dell'animazione. Ciò nonostante, la posizione della Chiesa riguardo all'aborto è rimasta sempre invariata; esso è stato sempre condannato con gravi pene stabilite o dai primi concili o dai Libri Penitenziali. E, anche se la penitenza prevista per il delitto di aborto procurato, con il passar del tempo è stata mutata e mitigata, non è stata cambiata la valutazione del delitto di aborto: è un omicidio e come tale deve essere punito, ma soltanto dopo che il feto si è già formato.

Quest'unanimità dei Padri della Chiesa, consensus Patrum riguardo all'aborto procurato, è obbligante per lo stesso Magistero della Chiesa, che non può mai derogare da ciò che la comunità cristiana ha sempre vissuto ed insegnato fin dalle stesse origini. Pochi temi hanno goduto di tanta unanimità nella vita della Chiesa dei primi secoli come questo. Si potrebbe, quindi, affermare che la difesa della vita del feto e la condanna dell'aborto costituiscono un assoluto teologico nell'insegnamento morale della Chiesa primitiva. Quest'insegnamento vige, perciò, anche nella Chiesa di oggi e la stessa Chiesa condanna questo „abominevole delitto" ${ }^{\text {" } 2}$ con la massima pena prevista nel Codex Iuris Canonici, cioè con la scomunica latae sententiae ${ }^{83}$.

motivi di pietà. Citando le norme precedenti, non riesce ad armonizzarle in modo soddisfacente, ancor meno riesce di essere chiaro mentre aggiunge alle antiche penitenze quelle ereditate dai penitenziali, dovendo qui distinguere tra il feto animato e quello non ancora animato, tra l'aborto a causa di povertà, e quello per occultamento di fornicazione precedente. Cf. J. Palazzini, Ius fetus ad vitam ciumcue tutela in fontibus ac doctrina canonica, op. cit., 98-107; B. Honings, Aborto e animazione ıntana, op. cit., 154-157; P. Sardi, L'aborto ieri e oggi, op. cit., 123-128.

81 Cf. Decretales Pseudo-Isidorianae et Capitula Angilramni, ed. P. Hinschius, Lipsiae 1863. 97 e 360.

82 Cf. Gaudium et spes 51.

83 Cf. can. 1398. Cf. anche A. Chrapkowski, Il delitto dell'aborto procurato nel Codex luris Canonici del 1917 e del 1983. Studio giuridico-storico, Roma 2001. 


\section{PRZESTEPSTWO ABORCJI \\ W NAUCZANIU KOŚCIOŁA PIERWSZYCH WIEKÓW}

(Omówienie)

Artykuł obejmuje okres od najstarszych dokumentów chrześcijańskich do początku średniowiecza, czyli od Ojców Apostolskich, poprzez nauczanie najbardziej znaczących pisarzy starochrześcijańskich, oficjalne wypowiedzi synodów aż do momentu wprowadzenia Ksiąg Penitencjalnych. W czasach Ojców Kościoła osąd moralny aborcji był zawsze negatywny. Pomimo wątpliwości co do momentu animacji płodu, oficjalna nauka Kościoła na temat aborcji pozostawała zawsze niezmienna, a samo przestępstwo było najsurowiej karane, o czym świadczą uchwały najstarszych synodów i surowe pokuty wyznaczane przez Księgi Penitencjalne.

Tego rodzaju consensus Patrum stanowi także dla dzisiejszego Magisterium Kościoła principium, od którego nie można odejść pod żadnym pozorem. Mało który z tematów moralnych w życiu Kościoła pierwszych wieków spotykał się z tak wielką jednomyślnością Ojców. Można więc twierdzić, że obrona poczętego życia ludzkiego i stanowczy sprzeciw wobec aborcji stanowiły jasną zasadę nauczania Kościoła pierwszych wieków. Także Kościół współczesny, kontynuując to nieprzerwane nauczanie, podkreśla wielkość przestępstwa aborcji i przewiduje za nie w swoim ustawodawstwie najwyższą karę, jaką jest ekskomunika latae sententiae. 